\title{
HOW CAN ONE POSSIBLY DETERMINE THE MULTIPLE INTELLIGENCES?
}

\author{
Sanda BORDEI, Ph.D., \\ "Alexandru Ioan Cuza" University of Iași, Romania \\ sanda.bordei@yahoo.com
}

\begin{abstract}
Measuring the multiple intelligences proves to be a very sensitive subject since Howard Gardner has always stated very clearly his position against the psychometric approach of the intelligence. This article aims to present some tools in the frame of the psychometric approach that could still be used in the every day practice of teaching and learning in order to identify and foster one s different types of intelligence. These are: Thomas Armstrong 's Multiple Intelligences Inventory, Multiple Intelligences Development Assessment Scale (MIDAS) for Children by Branton Shearer and McClellan \&Conti's Multiple Intelligences Inventory. They had been adapted for Romanian population for research purpose and the factor analysis was used. A discussion about the main findings and their limits will be presented.
\end{abstract}

Keywords: multiple intelligences, Gardner, MIDAS, factor analysis

\section{Theory of Multiple Intelligences in a Nutshell}

Theory of Multiple Intelligences was first presented by its author, Professor Howard Gardner, în 1983 in his book called Frames of Mind. The main assumption is that the intelligence is not seen as a single, static, monolythic construct but as a multiple and evolving one and very much depending on the social and cultural environment. Gardner defines it as a "biopsychological potential to process information that can be activated in a cultural setting to solve problems or create products that are of value in a culture" (Gardner, 2004, pp.60-61). So far he has identified and described eight types of intelligences: Linguistic, Logical-Mathematical, Spatial, Bodily-Kinesthetic, Musical, Interpersonal, Intrapersonal and Naturalistic and is still investigating the ninth one which would be the Existential Intelligence. Davis, Christodoulou., Seider \&Gardner(2011) offer the following short descriptions for each of them: "The Linguistic Intelligence represents the ability to analyze information and create products involving oral and written language such as speeches and books; Logical-Mathematical 
Intelligence is the ability to develop equations and proofs, make calculations, and solve abstract problems; Spatial Intelligence is the ability to recognize and manipulate large-scale and fine-grained spatial images; BodilyKinesthetic is the ability to use one's own body to create products or solve problems; Musical Intelligence is the ability to produce, remember, and make meaning of different patterns of sound; Interpersonal Intelligence is the ability to recognize and understand other people's moods, desires, motivations, and intentions; Intrapersonal Intelligence is the ability to recognize and understand his or her own moods, desires, motivations, and intentions and Naturalistic Intelligence is the ability to identify and distinguish among different types of plants, animals, and weather formations that are found in the natural world".

Gardner's studies proved that these intelligences are separately located in the brain, but they can be noticed to act together in the real life. And this is the reason why sometimes identifying them might result very challenging. For instance, graphs designing involves both spatial and logicalmathematical intelligence; or a pianist has a very well developed kinesthetic intelligence but also interpersonal and intrapersonal ones. As we know, Gardner declared himself completely against the psychometric approach of intelligence, arguing that the concept of IQ is very simplistic and far from reality as it is strongly de-contextualized and that besides the IQ defines only some of the intelligences, namely logical-mathematical, linguistic and spatial ones but has nothing to do with the others. And this is in fact the main or key attribute of his theory. Instead he supports the observation as the most powerful and appropriate method for establishing the cognitive profile as only watching somebody acting in various situations in the real life can provide the best information about the different levels of development of his/her intelligences at a certain moment. In the famous project Spectrum which first tested the multiple intelligences theory with 2 groups of kindergarten children, observation was the only one method used to identify the strengths and weaknesses in the cognitive profiles of the kids at a certain moment. The experimenters got very interesting results as they discovered many children who had various intelligences above average and neither their parents nor their teachers had succeeded in being aware of them before the project. We consider that this is the best proof for supporting the observation as the best approach in identifying the multiple intelligences, but at the same time we cannot stop remarking that the Spectrum project took place in very special conditions which would be ideal to find in the real world of education but which in practice still remain very rare, unfortunately. And that because the two experimental groups had a small number of kids each - up to 20, usually at least two adults who worked together with them, a friendly space 
full of various attractive didactic materials and no pressure at all regarding a fixed curriculum.

That is why although Gardner firmly stated his dislike for the psychometric approach, the pressure from teachers, parents and pupils determined various researchers to try to create some psychometric tools for identifying the multiple intelligences. The main reason is that on one hand as the observation is a very time consuming method, in the real life it is very difficult to be implemented on a large scale in schools or other formal and non-formal educational contexts and on the other hand because people tend to trust more this kind of tools than the "mere" observation. Besides, the large number of pupils in a classroom, the lack of diverse tools and appropriate materials to add value to the lessons, an unfriendly space full of barriers and the short time of a class prevent also the teachers from being able to observe their pupils in order to discover their cognitive profile so eventually a psychometric tool is needed to help them doing that.

At first glance, the great advantage of such a tool, if appropriate and scientifically built, would be that it would help the teachers, counselors and/or parents to be able to identify in very short time and with minimum of effort the cognitive profiles of their pupils. But at the same time the big challenge and even danger is that unconsciously people will tend to label once more the children as in their minds the questionnaire as a tool is closely connected with some label or categorization. In fact we could notice many times that people usually forget the core of Gardner's theory and make statements such as" this kid is musical or kinesthetic etc", completely forgetting that we all have more or less from all the 8 types of intelligences in our cognitive profile and, even more important, we are able to develop them all if we are determined and have or find a friendly environment.

So we perfectly understand and support this need for having a tool in order to measure the multiple intelligences, but at the same time we consider that it is crucial how we introduce this tool and how people are taught to use it in order to be in line with the ideas of Howard Gardner.

\section{Some Practical Aspects about Measuring the Multiple Intelligences}

We will present and discuss further in this article three different tools we have found so far that are used on a large scale to measure the multiple intelligences: the first one we will talk about is Thomas Armstrong's Multiple Intelligences Inventory, the second one is the Multiple Intelligences Development Assessment Scale (MIDAS) for Children created by Branton Shearer and the third one is McClellan \&Conti 's Multiple Intelligences Inventory. After a short description of each of them we will then run some factor analysis in order to check whether they present an appropriate factor structure in line with Gardner`s theory. 


\subsection{Thomas Armstrong `s Multiple Intelligences Inventory}

We chose to talk first about this tool as it seems to be very popular in the Romanian schools but also in various trainings of non-formal education such as one for Youthworkers we took part in several years ago. It is presented as a questionnaire with 79 dichotomic items which describe various daily life activities (e.g. I often like to spend my free time outdoors; Books are very important to me; I can easily compute numbers in my head etc.). The items in groups of 9 or 10 are being thought to underlie each of the 8 intelligences. In the end there is a specification to give one point for each "yes" answer and then to add them and find out the scores for each of the 8 intelligences and the biggest one indicates the dominant intelligence.

We were intrigued from the very beginning that we could not find any mention about its author nor about its basic psychometric properties such as validity and reliability. Then we started to check the Romanian literature related to Gardner's theory and finally we identified the source of this questionnaire as a teachers's training for differentiated instruction from the beginning of 2000 (for more details one can see Gliga\&Spiro,2001).

A real surprise came later when we found out by chance that in fact this tool had been translated from English and the source was Armstrong (1994), without being explicitely cited nowhere. But between the original and the translation there was one big difference the Romanian authors seemed to have completely neglected, namely that in the original source the tool was not meant to be a questionnaire, as Armstrong explicitely mentioned, but an inventory of activities, a kind of a checklistwith the aim to be only a starting point for discussion and reflection for growth and development and not at all for labelling! So the Romanian authors missed the esential and spread a tool which is not proper used at all and in fact collides with the core of Garner's theory itself! So it is that the starting point of the misusing of this tool which unfortunately spread very much up to the point that nowadays it became a very common instrument used to measure the multiple intelligences in our school.

We ran some factor analysis to check its factor structure on a database of 261 subjects but no interesting results could be find since from the very beginning the author's intention was not at all to propose a psychometric tool , as he clearly states in his book (Armstrong, 1994). The exploratory factor analysis was run using a tetrachoric correlation matrix since the answers are dichotomous. In order to generate the matrix we used the syntax code program TETRA-COM for estimation of the tetrachoric correlations (Lorenzo-Seva \&Ferrando, 2012). The results of the tetrachoric factor analysis run with this program showed $\mathrm{KMO}=.133$, Approx. Chi-Square= 3309.085, df. $=3828$, sig. $=1.000$, which means that the model is not a stable 
one. That is why we decided to do a tetrachoric factor analysis for each of the 8 intelligences separately as if they were independent factors. We then repeatedly excluded the items with low loadings on the factor and in the end we have got the following results:

For the spatial intelligence we have got $\mathrm{KMO}=.582$, Approx. ChiSquare $=312.594$, df. $=36$, sig. $=.000$, which means that the model is a stable one. The loadings of the items on factor are as follows: it 17: .251, it.26: .387 , it.32: .245 , it.39: .129, it.44: .131, it.47: .139, it.52: .382, it.58: .178 and it.63: .143. We have got three items with eigenvalues over 1 as follows: $2.051,1.748$ and 1.134 , which explain $54.809 \%$ of the variance of the spatial intelligence.

$\checkmark$ For the logical-mathematical intelligence we have got $\mathrm{KMO}=.787$, Approx. Chi-Square $=477.372$, df. $=21$, sig. $=.000$, which means that the model is a stable one. The loadings of the items on factor are as follows: it 11: .325 , it $20: .325$, it.34: .248 , it.37: .392, it.55: .358, it.64: .415 , it.68: .384 . We have got two items with eigenvalues over 1 as follows: 3.016 and 1.317 which explain $61.895 \%$ of the variance of the logical-mathematical intelligence.

$\checkmark \quad$ For the linguistic intelligence we have got $\mathrm{KMO}=.629$, Approx. ChiSquare $=200.714$, df. $=21$, sig. $=.000$, which means that the model is a stable one. The loadings of the items on factor are as follows: it 15: .112, it22: .138 , it.30: .207 , it.40: .196 , it.48: .175 , it.65: .324 , it.76: .125 . We have got two items with eigenvalues over 1 as follows: 2.036 and 1.391, which explain $48.954 \%$ of the variance of the linguistic intelligence.

$\checkmark$ For the interpersonal intelligence we have got $\mathrm{KMO}=.660$, Approx. Chi-Square $=500.595$, df. $=15$, sig. $=.000$, which means that the model is a stable one. The loadings of the items on factor are as follows: it 2: .324, it.25: .267, it.31: .348, it.56: .544, it.59: .535 and it.67: .551. We have got two items with eigenvalues over 1 as follows: 2.837 and 1.060 , which explain $64.950 \%$ of the variance of the interpersonal intelligence.

$\checkmark$ For the intrapersonal intelligence we have got $\mathrm{KMO}=.209$, Approx. Chi-Square $=217.758$, df. $=6$, sig. $=.000$, which means that the model is an unstable one. The loadings of the items on factor are as follows: it3: .400, it.36: .489, it.61: .383, it.77: .414. We have got three items with eigenvalues over 1 as follows: $1.482,1.228$ and 1.069 , which explain $94.478 \%$ of the variance of the intrapersonal intelligence.

$\checkmark \quad$ For the bodily-kinesthetic intelligence we have got $\mathrm{KMO}=.559$, Approx. Chi-Square $=252.891$, df. $=45$, sig. $=.000$, which means that the model is a stable one. The loadings of the items on factor are as follows: it 9: .074, it13: .167, it.29: .228, it.33: .111, it.43: .234, it.50: .147, it.57: .229, it.70: .204, it.73: .227 and it.79: .088. We have got four items with 
eigenvalues over 1 as follows: 1.972, 1.539, 1.274 and 1.062, which explain $58.467 \%$ of the variance of the bodily-kinesthetic intelligence.

For the musical intelligence we have got $\mathrm{KMO}=.827$, Approx. ChiSquare $=887.147$, df. $=36$, sig. $=.000$, which means that the model is a stable one. The loadings of the items on factor are as follows: it 7: .449, it 10: .357, it.14: .397 , it.23: .438 , it.27: .526 , it.38: .462 , it.46: .460 , it.62: .452 and it.74: .523. We have got two items with eigenvalues over 1 as follows: 4.091 and 1.228 , which explain $60.213 \%$ of the variance of the musical intelligence.

$\checkmark$ As for the naturalistic intelligence, we could not run any factor analysis because of an error message which said the matrix was not a positive one.

As we can see from the figures above, most of the items have very poor loadings on their factors which means that the tool the way it is now cannot be trusted for measuring the multiple intelligences.

\subsection{Multiple Intelligences Development Assessment Scale (MIDAS) for Children by Branton Shearer}

Unlike the previous tool discussed, this questionnaire is a psyhcometric one, very rigurous tested and in fact fully approved by Gardner, as he "considers Branton Shearer's efforts to measure multiple intelligences to be among the well-founded ones" (Shearer, 2013, pp.9). And this is exactly because

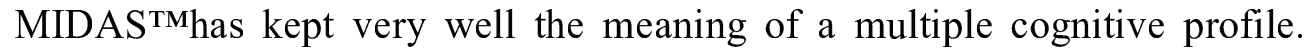
MIDAS $^{{ }^{T M}}$ does not mean at all another way of labeling persons but provides a useful image of how the cognitive profile of a person looks like at the moment of answering it and it is a very good start for in-depth analysis and personal development. The questionnaire has 93 self-reported items with Likert scale responses from $\mathrm{A}$ to $\mathrm{F}$ for kids from 10 to 14 years old. It is very easy to be filled in as the items are very practical and taken from the real life, being in fact a long enumeration of different activities one child is likely to do in his/her daily life. The tool proved to have good psychometric properties on a Romanian sample of 296 pupils from $6^{\text {th }}$ grade from urban and rural areas. A detailed description of the method and the presentation of the results can be find in Bordei (2015). In brief, we have got 6 factors out of 8 expected, namely Factor 1 which gathered the Personal Intelligences (interpersonal intelligence and intrapersonal intelligence), Factor 2 which gathered the Academic intelligences (logical-mathematical intelligence and linguistic intelligence), Factor 3 - Spatial intelligence, Factor 4 Kinesthetic Intelligence, Factor 5 - Naturalistic Intelligence and Factor 6 Musical Intelligence. The internal consistency remained high for each factor, alpha Cronbach values being: .921 for Factor 1, .828 for Factor 2, .831 for Factor 3, .796 for Factor 4, .854 for Factor 5 and .740 for Factor 6. Although 
this might seem not in line with the theory, until adulthood it is very normal to find the personal intelligences together as the Self is developing only inside of a social group and through the relationships with the others. Factor 2 needs indeed further research but still our findings are completely supported by the theory and the tool can be used in our culture.

The only problem with this tool might be that it is not free and even the cost per profile is only $1 \$$ it could be still not be accessible on a large scale.

\subsection{McClellan \&Conti s Multiple Intelligences Inventory}

In order to surpass the obstacle implied by the costs we checked the literature and found a free inventory to measure the multiple intelligences described by McClellan \& Conti (2008) but with no input by part of Howard Gardner. The authors set 27 items underlying 9 types of intelligences as they included also the Existential one. But since this intelligence has not been yet officially recognized by Gardner, we preferred to do an analysis on only the 24 items describing the 8 intelligences officially recognized by Gardner so far. The original tool was designed for adult learners but we chose to check it on a sample of 366 pupils from secondary school. So far we ran an exploratory factor analysis with Polymat-C program since the answers implied ranking (Lorenzo-Seva\& Ferrando, 2015). In order to extraxt the factors we used the unweighted least squares method and for the rotation matrix we used "Varimax" orthogonal method.

We have got $\mathrm{KMO}=.689$, Approx. Chi-Square $=2662,158$, df. $=276$, sig. $=.000$ which means that the data are good for the exploratory factor analysis.

We have got 12 factors with eigenvalues over 1 out of which the first 8 factors explain $53,41 \%$ din varianță. But when we took a closer look at the grouping of the items on each type of intelligence we noticed the following factor structure: $\mathrm{F} 1=$ Interpersonal intelligence (only the item 10 has a very good loading of .976, while item 18 has .064 and item 2 has .002), F2 = Intrapersonal intelligence (item $3=.385$ and item 19=.085), F3= Musical Intelligence (item $5=.378$, item $13=.243$ and item $21=.479$ ), $\mathrm{F} 4=$ Spatial Intelligence (item $8=.321$, item $16=.635$ and item $24=.502$ ), F5= Linguiestic Intelligence (item $7=.374$, item $15=.442$ and item $23=.003$ ), F6= Logicalmathematical Intelligence has no items with proper loading and that is why this factor could not be identifiedt, F7 = Naturalistic Intelligence (only item 6 $=.250$, the other two have low and negative loadings) and F8 = Bodilykinesthetic Intelligence (only item $9=.385$, the other two have low loadings).

\section{Discussion}

Midas- Kids and McClellan\&Conti questionnaires have been translated and then back translated as the procedure says by persons who knew English 
very well. The questionnaire inspired by Armstrong `s work has been taken as such from Gliga \& Spiro (2001) and 10 new items for the naturalistic intelligences have been added from an updated edition of Armstrong `s book. We mention that the first items for the naturalistic intelligence were most probably invented by the two authors and just added without any psychometric research beyond.

As we expected, we have found that the questionnaire inspired by Armstrong`s work cannot be trusted as a tool for measuring the multiple intelligences since the loadings on factors are in general very low. This is simply because this tool has never been meant to play such a role, which means that its use in our schools is completely wrong and without any foundation. The problem is that in our culture there is not any strong scientific culture developed yet and people just take everything they are given without checking by themselves the credibility of that source first before using it. That is why at this point we would kindly recommend a general statistics course at least for future teachers but also we appreciate it as being very valuable to start as soon as possible, even from the primary school. Among the benefits on one hand would be a shaping of the mind and on the other hand it would help everybody become more inquisitive and therefore active and independent.

As for the tool designed by McClellan and Conti, we were quite surprised to get such low loadings too. We can state that at this moment it cannot be used either in our culture for measuring the multiple intelligences. That is why for the moment we can recommend only Sharer's Midas -Kids as truly attaining its goal of identifying the multiple cognitive profile of a child between 10 and 14 years old. But in the near future we believe that it would be very interesting and also worthy to continue the research in this respect in order to create an appropriate tool that could be used safely in our schools. For this purpose we could also use the findings we have got so far and we can keep some items with good loadings as a starting point for this endeavor.

\section{Conclusions}

Measuring the multiple intelligences proves to be a very sensitive subject and the main danger is not to replace a label (IQ) with another (eight!) labels! As long as it is possible, the observation remains the best method to identify the multiple intelligences, ideally offering the subject (the pupils in our case) the chance to act in various sets and having at hand a lot of materials and stimuli. The practice proved that this is difficult to be obtained on a large scale and that is why various tools were created in order to measure the multiple intelligences even if this comes in contradiction with Howard Gardner's view against the psychometric approach because it is too rigid and de-contextualized. Since there is a understandable need for this 
kind of tools for the reasons we have already explained (time and trust) we believe that is extremely important to bear in mind that unlike the other psychometric tools (questionnaires, surveys and tests) in this case it is completely forbidden to label, since an intelligence that may be dominant at a certain moment in time may be replaced by other(s) that can become dominant if proper conditions are put in place. All we have to do with such an instrument is to use it as a starting point for further reflection and analysis, to transform its items as long as possible into in-depth interviews and to triangulate the data with other sources (products of the activity, impressions of important people for the subject who know him/her well etc). A research effort is also required for the future in order to adapt on the Romanian population or to create a reliable tool for identifying the multiple intelligences following of course the spirit of this fascinating theory.

\section{References}

Armstrong, Th. (1994). Multiple intelligences in the classroom.Alexandria, VA: ASCD

Bordei, S. (2015). MIDAS factor structure analysis for 12-14 y.o. Romanian pupils. Paper presented at the $6^{\text {th }}$ World Conference on Learning, Teaching and Educational Leadership, 29-31 October 2015, Paris, France.

Davis, K., Christodoulou, J., Seider, S., Gardner, H. (2011). The Theory of Multiple Intelligences. In: Sternberg $\mathrm{R} \mathrm{J}$, Kaufman $\mathrm{S}$ B Cambridge Handbook of Intelligence. New York: Cambridge University Press, 485503

Gardner, H. (2004). Frames of Mind. NY: Basic Books

Gliga, L., Spiro, J. coord. (2001). Instruirea diferenţiată. Aplicaţii ale teoriei inteligențelor multiple. Ghid pentru formatori şi cadre didactice.Bucureşti: Ministerul educaţiei şi cercetării

Lorenzo-Seva, U. , Ferrando, P. (2015). POLYMAT-C: A Comprehensive IBM SPSS program

for computing the polychoric correlation matrix. Behavior Research Methods 47, 884-889, doi 10.3758/s13428-014-0511-x

Lorenzo-Seva, U. , Ferrando, P. (2012).TETRA-COM: A Comprehensive SPSS program for

estimating the tetrachoric correlations. Behavior Research Methods 44, 1191-1196, doi 10.3758/s13428-012-0200-6

McClellan, J. A. \& Conti, G. J. (2008). Identifying the multiple intelligences of your students. Journal of Adult Education, 37(1), 13-38

Shearer, B. (2013). The MIDAS: A Professional Manual. USA 